\title{
Ant colony optimization algorithm based on mobile sink data collection in industrial wireless sensor networks
}

\author{
Hong Zhang ${ }^{1 *}$ (D), Zhanming $\mathrm{Li}^{1}$, Wanneng Shu ${ }^{2}$ and Jarong $\mathrm{Chou}^{3}$
}

\begin{abstract}
Industrial wireless sensor network (IWSN) has changed the information transmission way for existing industrial control system. In mobile sink-based industrial wireless sensor networks, the energy consumption optimization for data collection has always been a hot research issue. To meet the delay requirements and minimize energy consumption, a data collection strategy based on ant colony optimization with mobile sink is proposed for industrial wireless sensor networks. Firstly, in order to reduce the number of nodes directly accessed by sink and shorten the traversed path, the selection of rendezvous nodes based on entropy weight method is introduced according to the density of nodes, relative residual energy, and the degree of uniformity of distribution. Then, secondly, an ant colony optimization algorithm is proposed to obtain the optimal access path for mobile sink, which can achieve a trade-off between the energy consumption of the network and transmission delay. The simulation results show that, compared with the existing algorithms, the proposed algorithm can minimize the delay and prolong the lifetime of the network.
\end{abstract}

Keywords: Industrial wireless sensor networks, Delivery latency, Energy efficiency, Mobile sink

\section{Introduction}

Although the wireless sensor networks have been proposed, studied, and developed for more than a decade of years, there are still a lot of challenging issues especially in various industrial scenarios. Industrial wireless sensor networks (IWSN) is composed of autonomous sensor nodes distributed in monitoring area, which can acquire physical and environmental data such as temperature, sound, vibration, pressure, and movement. It has demonstrated enormous applicable value and commercial potential in aspect of military, industrial, and civil fields, such as, battlefield monitoring, industrial control process, machine operation, home automation, and traffic monitoring [1]. Due to limited battery power and laborious replacement of sensor nodes, energy efficiency is one of the most important issues in wireless sensor networks. With the scenario of static sink node, data forwarding in multi-hop manner will cause traffic concentration and make sensor

\footnotetext{
* Correspondence: 532337136@qq.com; zhh@gszy.edu.cn ${ }^{1}$ College of Electrical and Information Engineering, Lanzhou University Technology, Lanzhou 730050, People's Republic of China Full list of author information is available at the end of the article
}

nodes near the sink undertake more energy consumption. Hot-spot problem will worsen the network lifetime and even affect the operation of normal sensor nodes [2].

To deal with hot-spot problem, data collection using mobile sink in wireless sensor networks have been put forward in recent years. By using mobile sink node to travel in a certain path, it can reduce the energy consumption of sensor nodes and makes the energy consumption more balanced in the whole network [3]. Commonly, each node will be traversed by mobile sink node in turn, and the distance between them can be cut down to one hop. Then, the energy consumption for data forwarding will achieve the optimal resolution as well as high message delivery latency. For large-scale deployment, data collection efficiency will be very poor. A feasible solution is to tolerate a certain delay to compensate for the energy consumption and delay of data collection [4]. The whole network will be divided into several routing trees, and some sensor nodes act as rendezvous points. The other nodes forward the data to nearby rendezvous points, which can be traversed by mobile sink individually for data aggregation. Obviously, the mobile sink-based mode and path selection 
directly affect the efficiency of data collection and the overall performance of the network. Therefore, how to improve the energy utilization and collect as much data as possible with certain time delay constraint has become an important evaluation factor of the system. In this paper, we focus the network lifetime problem for time-sensitive data gathering and study the optimal visiting points and a data collection path for a mobile sink.

The rest of this paper is organized as follows. Section 2 shows the methods in industrial wireless sensor networks. Section 3 discusses the related work of data acquisition. Section 4 designs an optimal energy consumption model for industrial wireless sensor. The ant colony optimization algorithm is proposed in Section 5. Section 6 shows the simulation experimental results and concludes the paper with summary and future research directions.

\section{Methods}

Since the mobile sink-based mode and path selection directly affect the efficiency of data collection and the overall performance of the network, we performed this study to improve the energy utilization and collect as much data as possible with certain time delay constraint. To reduce the number of nodes directly accessed by sink and shorten the traversed path, the selection of rendezvous nodes based on entropy weight method is introduced according to the density of nodes, relative residual energy, and the degree of uniformity of distribution. Next, ant colony optimization is applied to obtain the optimal access path for mobile sink so as to achieve a trade-off between the energy consumption of the network and transmission delay.

\section{Related work}

Data collection is one of the most basic applications in industrial wireless sensor networks. When the node senses interesting events and collects data, it sends them to sink through single or multiple hops. Due to the energy constraints of sensor nodes, it is necessary to maximize the lifetime of the network on the basis of acquire efficient data delivery $[5,6]$. Accordingly, the trajectory of mobile sink may have a direct impact on the data delivery tasks in mobile sink-based data collection schemes. Sink mobility patterns in industrial wireless sensor networks can be classified as random mobility pattern, fixed-path mobility pattern, and controlled mobility pattern.

Recently, a number of data collection schemes have been proposed for mobile sink-based WSNs. Emre et al. [7] compared static and mobile data collection methods for sink nodes and establishes an optimization model of network lifetime. By being transformed into a linear model, the optimal solution of the trajectories of the mobile sink can be obtained. Guo et al. [8] proposed a converge-cast algorithm for efficient data collection in
MWSNs. The monitoring area is divided into several disks, and the rendezvous points (RPs) that are referred as specialized nodes working as sub-sinks will be chosen by employing quantum genetic algorithm. In [9], by conducting systematic analysis on the behaviors of the mobile sink in terms of both throughput capacity and lifetime of the network, Liu et al. presented a mobility-assisted data collection model and developed a comprehensive theoretical method to achieve beneficial throughput capacity and lifetime. Kumar et al. [10] proposed a clustering algorithm to divide all nodes into different clusters according to their location and employed classical traveling salesman algorithm to find the shortest path through all cluster centers. The time complexity of those algorithms increases sharply with the increase of the number of sensor nodes. Therefore, they are more suitable for scenarios with less number of sensor nodes and data transmission hops.

Other scholars study the selection method of sink node's mobile path in a distributed manner. Lee et al. [11] integrated the initial address, data collection routing, and dwell time of sink nodes to establish a linear programming model to obtain the optimal mobile sink sojourning pattern. Besides, by utilizing the variance of residual energy of neighboring sensor nodes, a simple practical heuristic algorithm for sink mobility is presented to alleviate the risk of disequilibrium of energy consumption among sensor nodes. Wang et al. [12] proposed a moving strategy called energy-aware sink relocation (EASR) for mobile sinks, which employs the information related to the node's residual energy and adjust the transmission range of sensor nodes and the relocating scheme for the sink adaptively. Wichmann et al. [13] employed the aircraft as a mobile sink node to collect the storage data of sensor nodes. According to its mobility characteristics, the mobile path based on TSP (traveling salesman problem) algorithm is reconstructed. In addition, the path is dynamically adjusted according to the estimated time of data collection to minimize the transmission delay. However, the algorithm does not consider the optimization of energy consumption of sensor nodes and network lifetime. Tashtarian et al. [14] established a network lifetime optimization model for data collection in a single-hop mode by using the location information of nodes and constructs a decision tree to solve the optimization model to obtain the mobile path of sink node. Ghosh et al. [15] decomposed the monitoring region into several triangulars, generating circles passing through three vertices of the triangle. Then, each circle center can be regarded as the RP. Sink node make use of greedy algorithm to determine the next residence location. However, due to the single-hop mode, the communication range of sink is limited. For the sake of data integrity of the whole monitoring region, it results in a longer mobile path and a higher data transmission delay. Shi et al. [16] proposed a 
routing protocol with dynamic layered to handle the overhead of mobile sink, which integrates Voronoi scoping and dynamic anchor selection to reduce the number of message interactions for updating routing tables.

The problem with sink mobility is concerned with the increase of data latency. Due to the mobility of sink nodes, some sensor nodes may have to wait for a long time consequently, which will cause the buffer overflow and increase the latency in data delivery. To achieve efficient data delivery to mobile sink, Kim et al. [17] designed an intelligent agent-based routing protocol for densely deployed and large wireless sensor networks. Pavithra et al. [18] proposed a weighted set programming algorithm based on rendezvous point (RP). By optimizing the data forwarding route from source node to fixed sink, the weight of each sensor node was calculated, and then the node was dynamically selected as the set point to realize that sink could traverse all the set points for efficient data collection. Wang et al. [19] proposed an optimal path construction method of variablelength coding parthenogenetic algorithm based on quadratic grid partitioning. Firstly, coarse-grained grids were used to partition the network area, and variable-length coding parthenogenetic algorithm was used to obtain the optimal path grids. Then, according to the initial optimal path constructed, fine-grained grids are used again for each passing grids to optimize the data collection path. Aiming at the problem of large delay caused by the speed constraints of mobile sink, Yu et al. [20] proposed a path optimization strategy. Based on the mathematical analysis of the relationship between delay and network energy consumption, a dynamically adjustable weight of Node's priority to data delivery is proposed. The optimal node weight is obtained by simulated annealing genetic algorithm, and the convergent node and the optimal mobile path finally can be resolved by multiple iterations.

However, none of the aforementioned approaches have considered minimization of both the stated objectives simultaneously. As this is an NP-hard problem [21], we are motivated to develop a data collection strategy based on ant colony optimization for wireless sensor networks with mobile sink.

\section{Proposed methods}

\subsection{System model}

Suppose that sensor nodes are randomly deployed in a rectangular monitoring area to form a multi-hop selforganizing network. The sensor network is fully connected, and all nodes have unique identities and are no longer mobile after deployment. Also, they are powered by limited energy and can not be supplemented. Each node should be configured with the same communication radius $r$ and has the same initial energy $E_{0}$. Sink node is capable of mobility and has external power supply. To reduce the high transmission overhead caused by longdistance transmission, the sensor node can forward data packets to the destination by multi-hop mode.

The sensor nodes can be classified into two kinds: rendezvous node $(\mathrm{RN})$ and ordinary node, and RNs can communicate directly with sink [22]. Comparatively, the ordinary node needs to send data to specific RN through a multi-hop mode. When sink node has not moved to the communication range, $\mathrm{RN}$ can put the aggregated data into the cache beforehand. Once the sink move along a certain trajectory and stay away with the RN within one hop, it can send to the sink directly. The first-order radio model is applied for energy depletion analysis, and the energy consumption in each round can be expressed as follows:

$$
E_{\text {total }}=\sum_{i=1}^{n}\left(e_{t r} k_{t}^{i}+e_{\text {rec }} k_{r}^{i}\right)
$$

In which, $e_{t r}$ and $e_{\text {rec }}$ represents the energy consumption for sending or receiving unit data. $k_{t}^{i}$ and $k_{r}^{i}$ represent the amount of data being received and sent by node $i$, respectively.

Assuming that the amount of data generated by each node in each round is $q$, there exists a relationship between the data received and the data forwarded by relay node $i$, i.e., $k_{t}^{i}=k_{r}^{i}+q$. Then, it can be deduced that the energy consumption of data transmission in the whole network is related to the number of hops during the data transmission, which can be given as

$$
\sum_{i=1}^{n} k_{r}^{i}=\sum_{i=1}^{n} h_{i} q
$$

where $h_{i}$ represents the minimum hop number that the node $i$ sends data to the fusion node.

Further, the total energy consumption can be expressed as the sum of the minimum hops among all sensor nodes, as shown in formula (3).

$$
\begin{aligned}
E_{\mathrm{total}} & =\sum_{i=1}^{n}\left(e_{t r} k_{t}^{i}+e_{\mathrm{rec}} k_{r}^{i}\right) \\
& =\sum_{i=1}^{n}\left[e_{t r}\left(k_{r}^{i}+q\right)+e_{\mathrm{rec}} k_{r}^{i}\right] \\
& =q\left[n e_{t r}+\sum_{i=1}^{n}\left(e_{t r}+e_{\mathrm{rec}}\right) k_{r}^{i}\right]
\end{aligned}
$$

It can be seen that optimization of the total energy consumption is equivalent to minimizing the sum of the hops from the ordinary nodes to fusion nodes. In the process of mobile sink's data collection, the number of hops depends on to the selection of rendezvous points of mobile sink. Therefore, the choice of sink mobile 
trajectory directly affects the energy consumption of the whole network [23].

\subsection{Data collection strategy with delay constraint}

According to the previous analysis, to achieve the delay requirements and minimize the overall energy consumption of the network, it is necessary to optimize the sink point trajectory in wireless sensor networks [24]. Furthermore, by considering delay constraints, the solution of optimal sink mobile path reduces the overall energy consumption. On the one hand, it is necessary to solve the optimal set of RNs to satisfy with the minimization the total energy consumption. On the other hand, the optimization of travel path selection by mobile sink needs to be fully considered to accord with the constraints for time delay [25].

For a given set of sensor nodes $S=\left\{s_{1}, s_{2}, \cdots, s_{n}\right\}$ and the set of RNs $V=\left\{v_{1}, v_{2}, \cdots, v_{K}\right\}$, an undirected graph $G=\{\Psi, E\}$ can be constructed with a collection of edges $E$, and $\Psi=S \cup V$. The problem of data collection with mobile sink can be transformed into Traveling Salesman Problem. By constructing the path set $\operatorname{PATH}(G)$ for data collection based on the undirected graphs $G$, it will ensure that the hops from any ordinary node to RN can not exceed the limit value $h_{\max }$ as well as obtaining the minimization of the total energy consumption.

Let $P_{i}=\left\{p_{i, 1}, p_{i, 2}, \cdots, p_{i, k_{i}}\right\}$ denote the set of all paths from the sensor node $i$ to its adjacent RN node set, in which $p_{i, j}$ is the $j$-th path from $k_{i}$ data forwarding paths. Therefore, the location selection of RNs can be formulated as an optimization problem:

$$
\begin{aligned}
& \min _{P \in P_{s}(G)} E_{\text {total }}(P) \\
& \text { s.t. } \quad \forall P_{i} \in P A T H, \exists p_{i, j} \in P A T H, \operatorname{dist}(i, j)+\operatorname{dist}(j, v(i)) \leq h_{\max } r \\
& \max _{i=1,2, \cdots, n}\{h(i, v(i))\} \leq h_{\max } 1 \leq i \leq n, 1 \leq j \leq\left|P_{i}\right|
\end{aligned}
$$

where $\operatorname{dist}(i, j)$ is the Euclidean distance between node $i$ and $j . v(i)$ is the next-hop RN being chosen by node $i$, and the number of the corresponding hops can be defined by $h(i, v(i))$. Besides, $r$ indicates the communication radius of the sensor node.

For any node $j$ on the path $P_{i}$, the sum of the hops to the access path does not exceed the threshold of $h_{\max }$. Since the Euclidean distance between two adjacent nodes is less than the communication radius $r$, the sum of the distance from node $j$ to $v(i)$ and the distance between node $j$ and $i$ can be no more than $h_{\max } r$.

\subsection{Determination of rendezvous points}

In order to ensure the overall energy depletion of the network being balanced, the selection of resident points may take into account comprehensively about the factors, including the node's density, relative residual energy, and uniformity of distribution. As a regional center directly interacts with sink, $\mathrm{RN}$ is responsible for data fusion of other nodes within a certain range and has to undertake more energy consumption [26, 27]. The ratio of residual energy to initial energy of a node is defined as relative energy. In order to achieve better energy consumption balance, the larger the relative energy is, the node can be more competitive. In addition, the node density is defined as the number of nodes in the communication range. The higher the node density, the lower the average energy depletion of nodes will demonstrate during the course of inter-cluster communication. By taking into account of the balanced distribution of RNs in the whole network, it is beneficial to improve the overall transmission efficiency of the network. Therefore, the nodes with larger centroid degree, i.e., the degree of proximity between a node and its neighbors, should be selected as far as possible. Based on the above analysis, the relevant indicators are defined as follows:

$$
\left\{\begin{array}{l}
f_{1}=\frac{E_{\text {res }}(i)}{E_{0}} \\
f_{2}=\frac{\sum_{j \in \text { neighbor }(i)} \operatorname{cov}(i)}{\operatorname{cov}(j)} \\
f_{3}=\frac{D(i)}{\max \{D(i)\}} \\
D_{i}=1-\frac{\sqrt{\left(x_{i}-\frac{1}{n} \sum_{j=1}^{n} x_{j}\right)^{2}+\left(y_{i}-\frac{1}{n} \sum_{j=1}^{n} y_{j}\right)^{2}}}{r}
\end{array}\right.
$$

where $E_{\text {res }}(i)$ represents the residual energy of node $i$ and $E_{0}$ is the initial energy. $\operatorname{cov}(i)$ denotes the number of nodes within the communication radius of node $i$, and neighbor $(i)$ represents the neighbor nodes set. In addition, $D(i)$ is defined as the centroid degree of node $i$.

The weight of each indicator is determined by entropy weight method. First of all, normalize the above indicators as:

$$
\tilde{f}_{m}=\frac{f_{m}(i)-\min \left\{f_{m}(i)\right\}}{\max \left\{f_{m}(i)\right\}-\min \left\{f_{m}(i)\right\}}
$$

where $\max \left\{f_{m}(i)\right\}$ and $\min \left\{f_{m}(i)\right\}$ denotes the original minimum and maximum value of the index function, respectively. $f_{m}(i)$ is the $m$-th index function of node $i$.

Next, the group vector entropy and weight values can be calculated as: 


$$
\left\{\begin{array}{l}
U_{m^{-}}-\frac{1}{\ln K} \sum_{1-U_{m}} \tilde{f}_{m}(i) \ln \tilde{f}_{m}(i) \\
w_{m}=\frac{\left.1-U_{m}\right)}{\sum\left(1-U_{m}\right)}
\end{array}\right.
$$

According to the index function value and the weight value, the comprehensive evaluation of each node can be determined by formula (8). The sensor node with higher value will be chosen as the candidate resident point.

$$
\text { Cand_RN } R(i)=\sum w_{m} \tilde{f}_{m}(i)
$$

\subsection{Path selection based on ant colony optimization}

Ant colony optimization (ACO) is a kind of bionic technique inspired by the behavior of real ants from nature [28]. By finding optimal paths through graphs, it demonstrates good enough performance for solving combinatorial optimization problems and near-optimal solutions to the travelling salesman problem. Basically, ant colony optimization algorithm is a kind of positive feedback mechanism, which has the characteristics of randomness, adaptability, and distribution. It is suitable for parallel computation and accurate solution [29]. However, there are two disadvantages in traditional ACO algorithms. One is the operational inefficiency owing to the diversity of ants, the other is that it is easy to fall into local convergence and the obtained path usually can not meet with the optimal solution [30].

When sink obtains the set of RNs $\Psi=\left\{v_{1}, v_{2}, \cdots, v_{K}\right\}$, the planning of the travel path can be approximated to TSP problem, which can be resolved approximately by adopting ant colony algorithm. During the course of the current node ants choosing the next-hop node from the neighboring node set, the probability of selection is crucial. Denote $\tau_{i j}(t)$ as the pheromone concentration on the path from node $i$ to $j$. The heuristic function $\eta_{i j}(t)$ can be defined as: $\eta_{i j}(t)=1 /|d(i, \sin k)-d(j, \sin k)|$, which indicates the expected degree of ants from $i$ to $j . d(i$, sink) and $d(j, \operatorname{sink})$ represent the distance from the mobile sink to either the current RN or the next one, respectively. $N_{k_{a}}$ denotes the set of RNs to be visited by ant $k_{a}$. In addition, $\alpha, \beta$ are parameters to regulate the relative weight of the pheromone trail and heuristic value, which can be satisfied with $\alpha \geq 0, \beta \geq 0, \alpha+\beta=1$. Hence, the probability of moving from RN $i$ to $j$ can be expressed as follows:

$$
p_{i j}(t)=\left\{\begin{array}{l}
\frac{\left[\tau_{i j}(t)\right]^{\alpha} \cdot\left[\eta_{i j}(t)\right]^{\beta}}{\sum_{u \in N_{K i}}\left[\tau_{i u}(t)\right]^{\alpha} \cdot\left[\eta_{i u}(t)\right]^{\beta}}, k \in N_{i} \\
0, \quad \text { other }
\end{array}\right.
$$

For the distance constraint, the gain of the access points set with minimum energy consumption is obviously optimal. Therefore, it is reasonable to choose the access node based on the energy cost and distance from mobile sink. By defining the effect of RN selection on global energy consumption in utility priority metric path, we introduce the concept of path superiority as a criterion for ant path evaluation after a round. The smaller the superiority is, the better the path quality will be. Then, the path superiority can be defined as:

$$
\begin{aligned}
P R(i)= & \lambda_{1} \frac{E\left(V_{k_{a}} \cup\left\{v_{i}\right\}\right)-E\left(V_{k_{a}}\right)}{\operatorname{TSP}\left(V_{k_{a}} \cup\left\{v_{i}\right\}\right)-L\left(V_{k_{a}}\right)}+\lambda_{2} \frac{E\left(V_{k_{a}}\right)-E\left(V_{k_{a}}-\left\{v^{\prime}\right\}\right)}{L\left(V_{k_{a}}\right)-T S P\left(V_{k_{a}}-\left\{v^{\prime}\right\}\right)}, \\
& \operatorname{TSP}\left(V_{k_{a}} \cup\left\{v_{i}\right\}\right) \leq D_{m} V_{m}
\end{aligned}
$$

In which, $V_{k_{a}}$ represents the $\mathrm{RN}$ set being visited, $L\left(V_{k_{a}}\right)$ indicates the corresponding shortest path length, and $E\left(V_{k_{a}}\right)$ represents the total energy consumption for transferring data to RNs.

According to the ratio between energy gain and distance cost, path superiority can evaluate the influence of global optimal solution for candidate node to be selected as next hop. Then, the determination of the relative can impact on the pheromone and the heuristic for the decision of the ant.

Furthermore, after the ants end their tours, the pheromone trail amount on every edge $(i, j)$ is updated by

$$
\tau_{i j}(t+1)=(1-\rho) \tau_{i j}+\rho \Delta \tau_{i j}(t)
$$

where $\rho$ is the local pheromone decay parameter and $\Delta \tau_{i j}(t)$ is the added pheromone trail amount at the point $t$.

The forward ants move from access point $i$ to $j$ for pheromone updating, and the updating rules are as follows:

$$
\Delta \tau_{i j}(t)=\frac{\left.Q_{L} E(j)\right)}{E(i)+d(i, j)}
$$

where $Q_{L}$ represent the local pheromone concentration. $E(i)$ and $E(j)$ represent the energy consumption for data being migrated from the member nodes to $\mathrm{RN} i$ and $j$, respectively. $d(i, j)$ is the Euclidian distance.

Subsequently, the backward ants go back to the source along the reverse path and complete global pheromone updating, and the corresponding updating rules can be given as

$$
\Delta \tau_{i j}^{k_{a}}(t)=\frac{Q_{G}}{\operatorname{PATH}\left(G, k_{a}\right) * P R(i)}
$$

where $Q_{G}$ represents the Global pheromone concentration and $\operatorname{PATH}\left(G, k_{a}\right)$ is the path length of the ant $k_{a}$.

Formula (13) shows that updating pheromones through path superiority ensures the energy requirement of the 


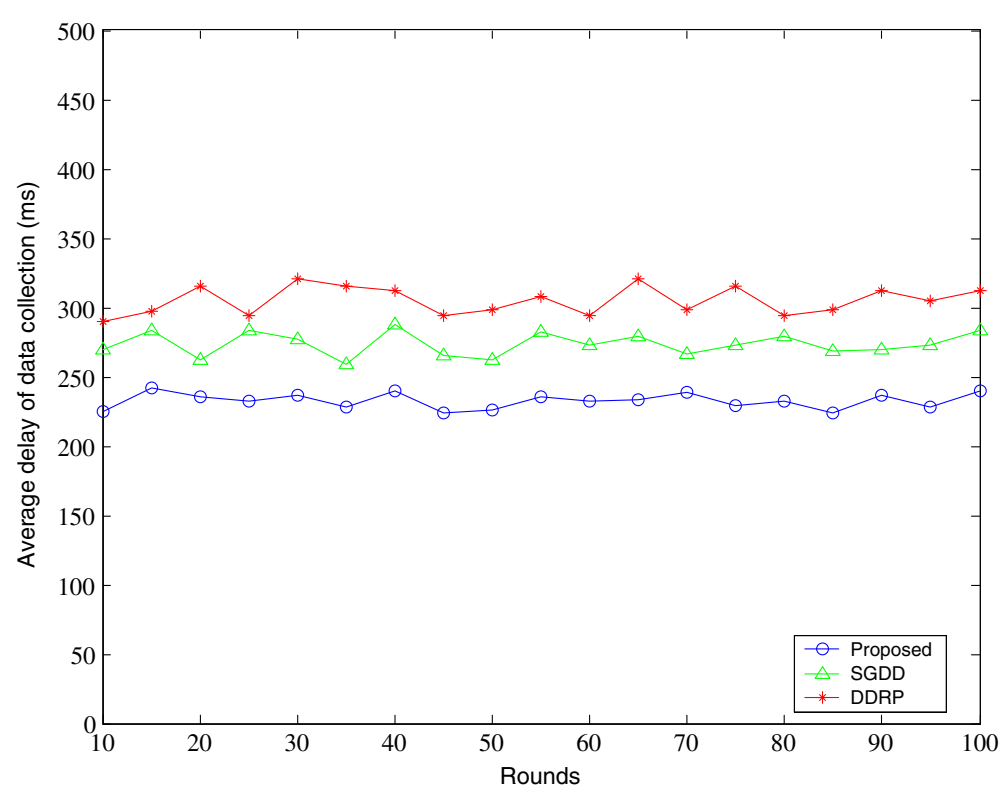

a) 200 sensor nodes

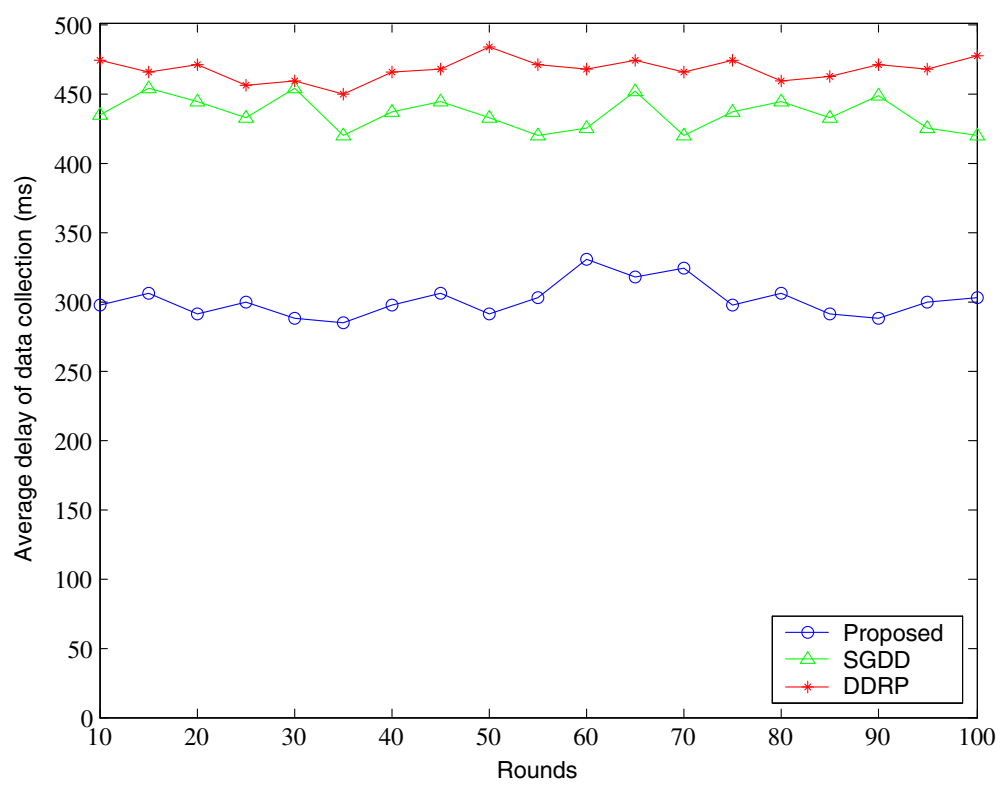

b) 400 sensor nodes

Fig. 1 The average delay of data collection

path and takes into account the energy balance, which is conducive to improving the efficiency of data collection.

\section{Experimental results and analysis}

In this section, several numbers of simulations are conducted and evaluated to verify the performance of our proposed method. The main parameters of the environment are as follows: the sensor nodes are randomly deployed in a rectangular monitoring area of $200 \times 200 \mathrm{~m}$, the initial energy of all sensor nodes is $2 \mathrm{~J}$. The communication radius is equal to $50 \mathrm{~m}$, and the delay requirement is set as $10 \mathrm{~min}$. The energy consumption of transmitting and receiving circuits in the energy consumption model is set to $50 \mathrm{~nJ} / \mathrm{bit}$, and the moving speed of mobile sink is fixed to $2 \mathrm{~m} / \mathrm{s}$. Besides, $\lambda_{1}$ and $\lambda_{2}$ is equal to 0.5 . The proposed method is compared with related works, data-driven routing protocol 


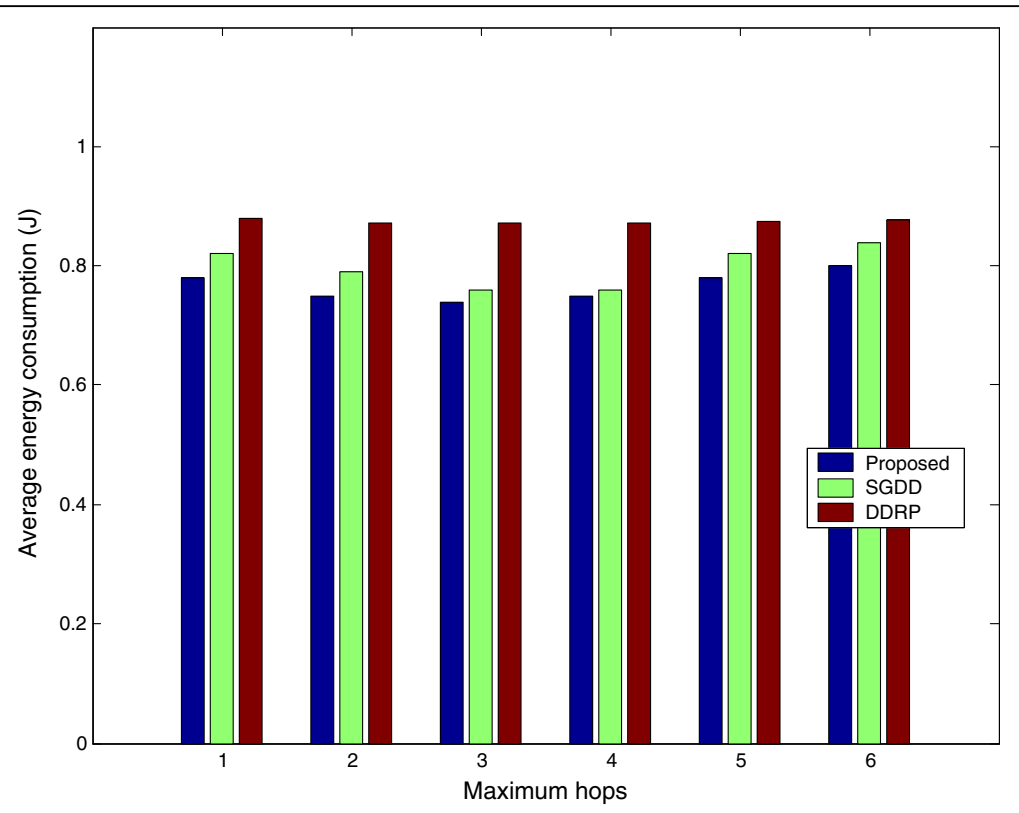

a) 200 sensor nodes

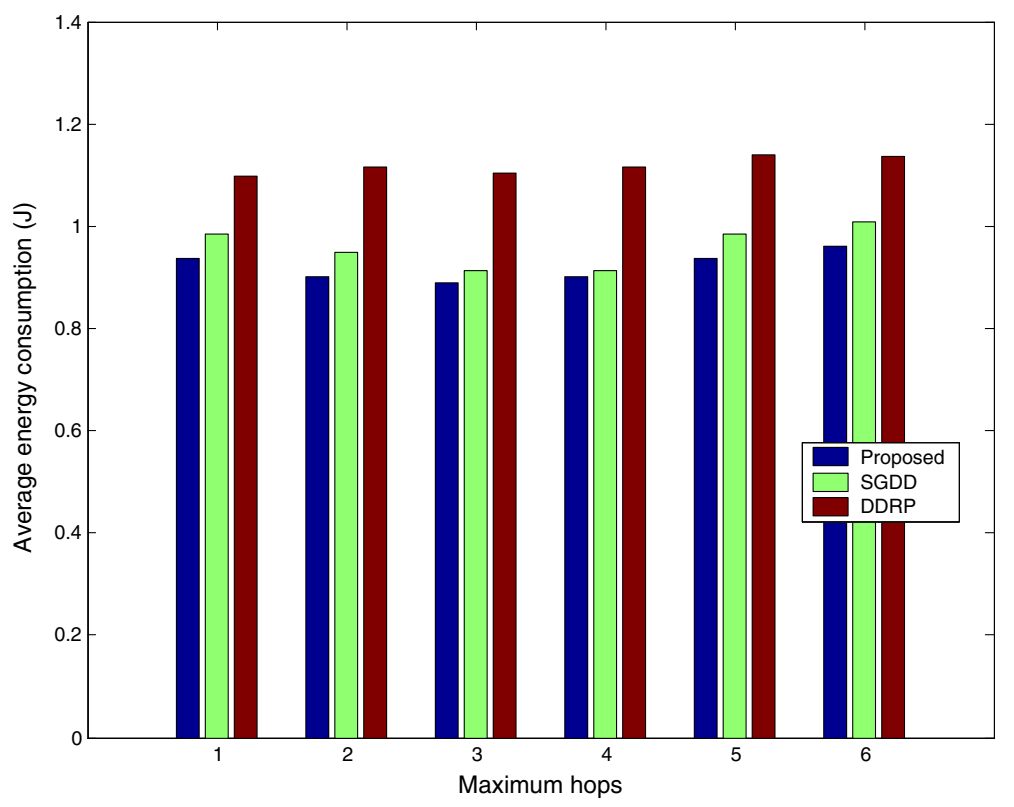

b) 400 sensor nodes

Fig. 2 The average energy consumption under different hop limits

(DDRP) [31] and self-managed grid-based data dissemination protocol (SGDD) [32], for data aggregation with mobile sink.

Figure 1 shows the comparisons of the average delay of data collection in mobile sink mode. In order to reflect the global performance and avoid the impact of energyexhausted nodes in advance, the rounds without a dead node is selected. From the experimental results, it can be seen that DDRP takes the longest time to complete a cycle of data acquisition, followed by SGDD and proposed method. The reason is that DDRP needs more nodes to access directly and the relay nodes in the network are dispersed, which bring about the transmission of data over long distance. In the proposed method, the priority of RN nodes can be defined to optimize the travel path of a mobile sink. The data collection efficiency of sink is improved and the data collecting latency can be reduced more sharply than other algorithms. Especially, when the 


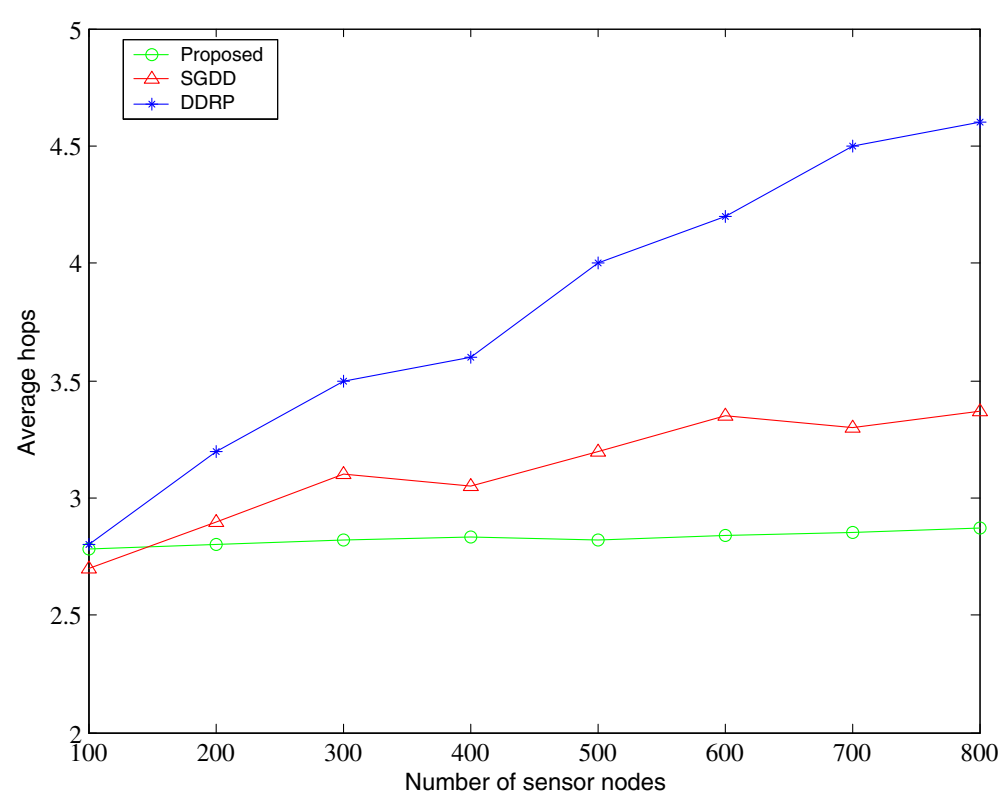

Fig. 3 The average number of hops

nodes are distributed densely, the difference of average delay is more obvious.

Figure 2 shows the comparisons of the average energy consumption of the three algorithms under different hop limits. Basically, when the maximum hop number is set as 3 or 4, SGDD and PROPOSED can achieve the optimal average energy consumption. It demonstrates that the overall energy consumption of the network can not reach the optimal state under the condition of single hop mode and excessive hops. This is because by employing irregular clustering, and the long forwarding path from some subnodes to the sink node in SGDD, will lead to a significant increase in aspect of the overall energy consumption when the number of hops increases. Similarly, if setting the maximum hops without restraint, it will lead to excessive energy consumption of RNs for data aggregation and be not conducive to the minimization of average energy consumption. Comparatively, the average energy consumption is not sensitive to maximum hop constraints in DDRP. However, the experimental results clearly show

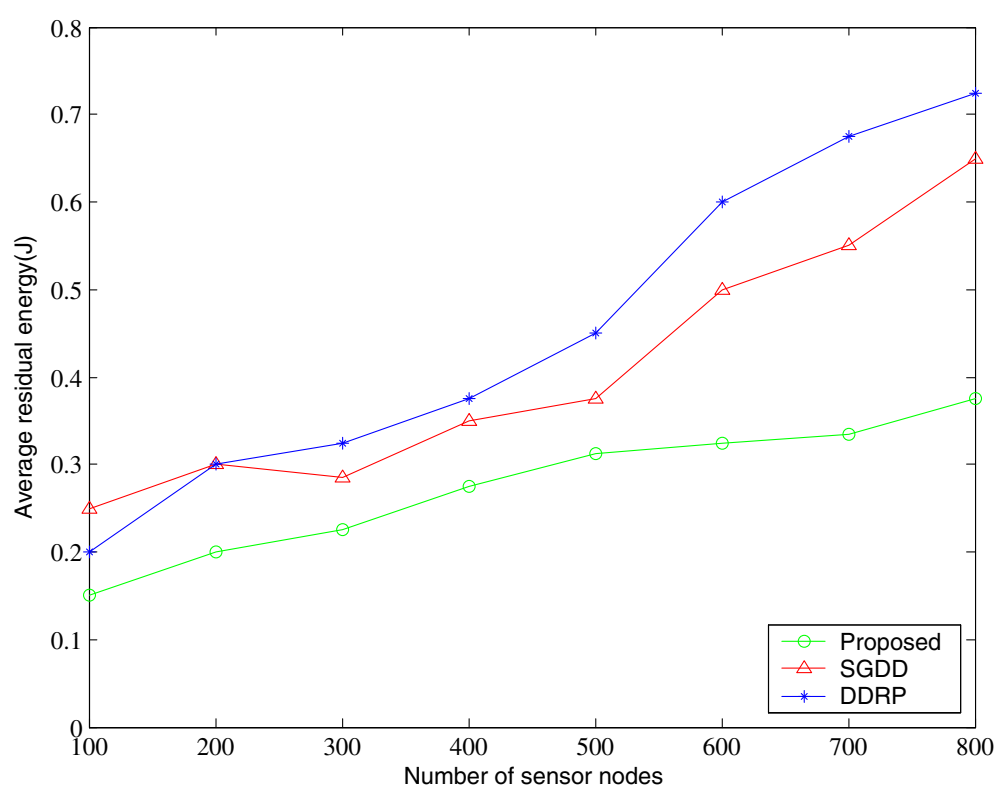

Fig. 4 The average residual energy 


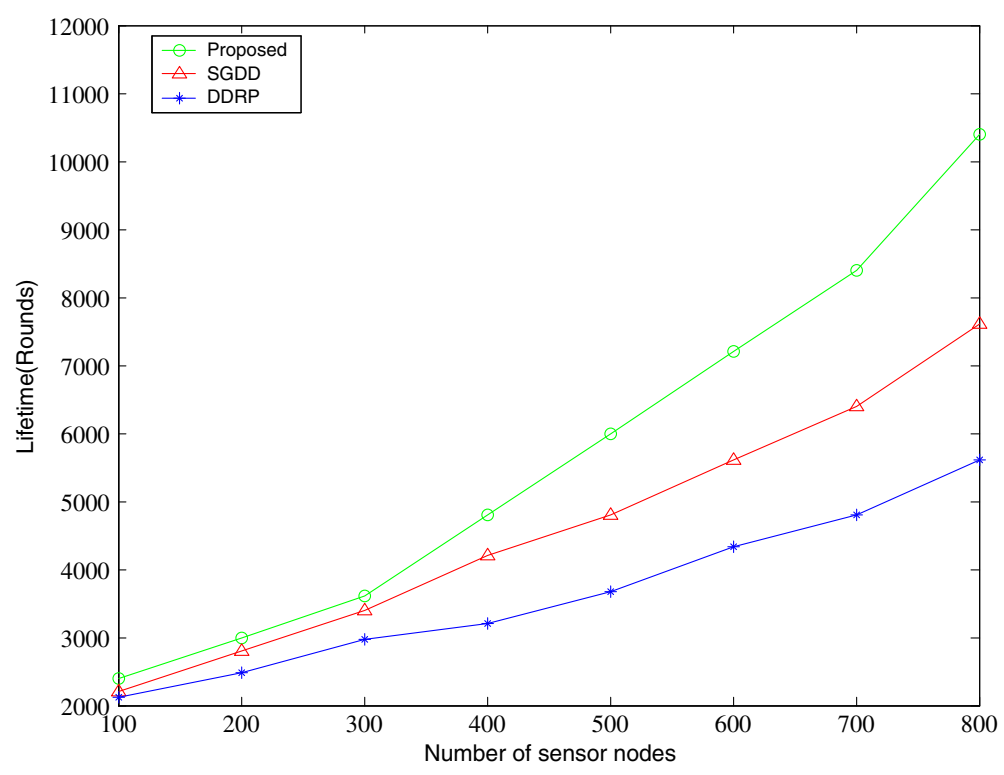

Fig. 5 Network lifetime

that the average energy consumption of DDRP is much higher than that of the other two algorithms.

Next, to verify the effectiveness of the proposed algorithm, its performance is compared with DDRP [31] and SGDD [32] in terms of the average hops, average residual energy, and network life time for different numbers of sensor nodes. Figure 3 illustrates the average number of hops from normal nodes to mobile sink for different numbers of sensor nodes. As it can be seen from the result, the average hops from source node to mobile sink demonstrates less variation by our proposed method, which is beneficial from a set measure used in multi-objective optimization to estimate the RNs. SGDD takes the nearest node from mobile sink as the root node to dynamically construct the number of routes, and lacks of global consideration, which affects the stability of average hops. By utilizing a random clustering method rather than probabilistic model to weaken the impact of "hot spot" problem, it makes DDRP's performance lag behind other algorithms.

Figure 4 illustrates the average residual energy of nodes when network ends. When the network is no longer able to support normal task, the average residual energy of surviving nodes reflects the utilization of node resources and whether the energy consumption of nodes is balanced in the process of operation. By considering the residual energy of candidate nodes, our proposed method handle the nodes near the resident point with priority and make the mobile sink change its moving trajectory to balance the energy consumption of the nodes. In contrast, the cluster heads usually are far away from the mobile sink in DDRP, which affects the energy balance of the network negatively.

In Fig. 5, we compare the network lifetime for different numbers of sensor nodes. The network lifetime in this test is defined as the time when the first node runs out of its energy. From the simulation results, the lifetime of our proposed method can achieve higher than that of both SGDD and DDRP in all cases. The reason behind the increased network lifetime manifests the highly available data gathering strategy and moving trajectory strategy with ACO optimization, which can result in minimum energy consumption and better load balancing among the sensor nodes. In DDRP, the mobile sink dwelled more time, which caused the nodes near the mobile sink to waste higher energy consumption and die prematurely.

\section{Conclusions and future work}

The oncoming requirement on the industrial wireless sensor networks covers from the lower physics layers to the upper application layer. In this paper, we propose an ant colony optimization algorithm based on mobile sink data collection in industrial wireless sensor networks. To reduce the number of nodes directly accessed by sink and shorten the traversed path, the selection of RNs based on entropy weight method is introduced according to the density of nodes, relative residual energy, and the degree of uniformity of distribution. Furthermore, an ant colony optimization algorithm is introduced to obtain the optimal access path for mobile sink, which can achieve a trade-off between the energy consumption 
of the network and transmission delay. The simulation results show that, compared with the existing algorithms, the proposed algorithm can minimize the delay and prolong the lifetime of the network.

In our future research, we will try to optimize the scheme in a scenario with obstacles, and design a complete protocol for communication for data collection with mobile sink in industrial wireless sensor networks.

\section{Abbreviations}

ACO: Ant colony optimization; DDRP: An efficient data-driven routing protocol; IWSN: Industrial wireless sensor networks; RN: Rendezvous node; RP: Rendezvous point; SGDD: Self-managed grid-based data dissemination protocol; TSP: Traveling salesman problem

\section{Acknowledgements}

The authors acknowledged the anonymous reviewers and editors for their efforts in valuable comments and suggestions.

\section{Authors' contributions}

$\mathrm{HZ}$ proposed the innovation ideas and theoretical analysis, and ZL carried out the experiments and data analysis. WS conceived of the study, participated in its design and coordination, and helped to draft the manuscript. All authors read and approved the final manuscript.

\section{Funding}

This work was supported by the Project of Gansu Province for Industrial and Information Development (Grant No.23051358), Gansu Province for Guiding Scientific and Technological Innovation and Development (Grant No. 2018ZX-05), and National Natural Science Foundation of China (Grant No. 61603420

\section{Availability of data and materials}

Data sharing is not applicable to this article as no datasets were generated or analyzed during the current study.

\section{Competing interests}

The authors declare that they have no competing interests.

\section{Author details}

${ }^{1}$ College of Electrical and Information Engineering, Lanzhou University Technology, Lanzhou 730050, People's Republic of China. ${ }^{2}$ College of Computer Science, South-Central University for Nationalities, Wuhan 430074, People's Republic of China. ${ }^{3}$ Deparment of Electrical and Computer Engineering, Michigan State University, East Lansing, MI 48824, USA

Received: 15 February 2019 Accepted: 15 May 2019

Published online: 10 June 2019

\section{References}

1. C. Tunca, S. Isik, M.Y. Donmez, C. Ersoy, IEEE Commun. Surv. Tutor. 16(2), 877-897 (2014)

2. S. Yang, U. Adeel, Y. Tahir, J.A. McCann, IEEE Trans. Mob. Comput. 16(5), 1420-1433 (2017)

3. N. Ghosh, R. Sett, I. Banerjee, Comput. Electr. Eng. 64, 288-304 (2017)

4. Z. Huang, G. Shan, J. Cheng, J. Sun, Neural Comput. Appl. (2018). https://doi. org/10.1007/s00521-018-3728-2

5. F. Tashtarian, M. Hossein, Y. Moghaddam, K. Sohraby, S. Effati, IEEE Trans. Veh. Technol. 64(7), 3177-3189 (2015)

6. M.E. Keskin, I.K. Altinel, N. Aras, Comput. J. 54(12): 1987-1999 (2011).

7. M.E. Keskin, I.K. Altinel, N. Aras, C. Ersoy, Comput. J. 54(12), 1987-1999 (2011)

8. J. Guo, L.J. Sun, W.J. Xu, J. Commun. 33(9), 176-184 (2012)

9. W. Liu, K. Lu, J. Wang, G. Xing, L. Huang, IEEE Trans. Veh. Technol. 61(6), 2777-2788 (2012)

10. A.K. Kumar, K.M. Sivalingam, A. Kumar, Wirel. Netw 19(3), 285-299 (2013)

11. K. Lee, Y.-H. Kim, H.-J. Kim, S. Han, Wirel. Netw 20(2), 303-318 (2014)

12. C.-F. Wang, J.-D. Shih, B.-H. Pan, T.-Y. Wu, IEEE Sensors J. 14(6), 1932-1943 (2014)

13. A. Wichmann, T. Korkmaz, Comput. Commun. 72(72), 93-106 (2015)

14. F. Tashtarian, M.H.Y. Moghaddam, K Sohraby, S. Effati, Comput. Netw. 77, 128-143 (2015)
15. N. Ghosh, I. Banerjee, Comput. Electrical Engin. 48(10), 417-435 (2015)

16. L. Shi, Z. Yao, B. Zhang, C. Li, J. Ma, Int. J. Commun. Syst. 28(11), 1789-1804 (2015)

17. J.W. Kim, J.S. In, K. Hur, J.W. Kim, Consumer Electron. IEEE Trans. 56(4), 2310-2316 (2010)

18. H. Pavithra, R. Shivashankar, G.R. Poornima, in Proceedings of the 2016 IEEE International Conference on Recent Trends in Electronics Information Communication Technology (IEEE, Piscataway, 2016), pp. 151-155

19. W. Wang, H.S. Shi, P.Y. Huang, B.J. Gao, J.P. Niu, J. Wang, J. Northwestern Polytechnical Univ. 34(6), 1016-1021 (2016)

20. Z.B. Yu, X.X. Kong, J.J. Pei, Transducer Micro-system Technol. 35(11), 44-46 (2016)

21. A. Somasundara, A. Ramamoorthy, M. Srivastava, IEEE Trans. Mob. Comput. 6(4), 395-410 (2007)

22. B. Suh, S. Berber, Electron. Lett. 52(2), 167-169 (2015)

23. F. Tashtarian, M. Hossein, Y. Moghaddam, K. Sohraby, S. Effati, IEEE Trans. Veh. Technol. 64(7), 3177-3189 (2015)

24. A.W. Khan, A.H. Abdullah, M.H. Anisi, J.I. Bangash, Sensors 14, 2510-2548 (2014)

25. A. Kaswan, P.K. Jana, M. Azharuddin, In proceedings of advances in computing, communications and informatics, ICACCl, 2017 International Conference on IEEE (2017), pp. 168-173

26. L. Danpu, Z. Kailin, D. Jie, China Commun. 10(3), 114-123 (2013)

27. R. Logambigai, A. Kannan, Wirel. Netw 22, 945-957 (2016)

28. M. Dorigo, L.M. Gambardella, IEEE Trans. Evol. Comput. 1(1), 53-66 (1997)

29. L.M. Gambardella, É.D. Taillard, M. Dorigo, J. Oper. Res. Soc. 50(2), 167-176 (1999)

30. J.W. Lee, B.S. Choi, J.J. Lee, IEEE Trans. Ind. Inf. 7(3), 419-427 (2011)

31. M.R. Majma, S. Almassi, H. Shokrzadeh, Int. J. Commun. Syst. 29, 959 (2016)

32. L. Shi, B. Zhang, K. Huang, J. Ma, Int. J. Commun. Syst. 26(10), 1341-1355 (2012)

\section{Publisher's Note}

Springer Nature remains neutral with regard to jurisdictional claims in published maps and institutional affiliations.

\section{Submit your manuscript to a SpringerOpen ${ }^{\circ}$ journal and benefit from:}

- Convenient online submission

- Rigorous peer review

- Open access: articles freely available online

- High visibility within the field

- Retaining the copyright to your article

Submit your next manuscript at $\boldsymbol{\nabla}$ springeropen.com 